\title{
Targeted next generation sequencing of breast implant- associated anaplastic large cell lymphoma reveals mutations in JAK/STAT signalling pathway genes, TP53 and DNMT3A
}

Breast implant-associated anaplastic large cell lymphoma (BI-ALCL) is an uncommon neoplasm occurring in women with either cosmetic or reconstructive breast implants (Clemens et al, 2016). Until now, most studies have focused on defining the clinico-pathological features of BI-ALCL, leading to its inclusion as a new provisional entity, a subtype of anaplastic lymphoma kinase (ALK)-negative ALCL, in the revised World Health Organization classification of lymphoid malignancies (Swerdlow et al, 2016). BI-ALCL is characterized by the presence of $\mathrm{CD} 30^{+}$large atypical lymphocytes frequently confined to the peri-implant seroma fluid. Nevertheless, solid infiltrating masses and cases pursuing an aggressive clinical course have been reported. The surgical and pathological staging system designed by Clemens et al (2016) suggests that BI-ALCL has a pattern of progression similar to that of solid tumours rather than non-Hodgkin lymphomas, and that the effusion- and solid-types might represent different stages of the same disease rather than two distinct variants.

The molecular pathogenesis and mechanisms of progression of BI-ALCL, however, remain largely unknown, thus limiting the identification of biomarkers that enable disease prognostication and optimal treatment. Hence, we performed targeted next generation sequencing of seven BI-ALCL, identified in the archives of three institutions over 7 years, to investigate the presence of underlying somatic mutations. Informed consent was obtained from patients and the study was performed in accordance with the Declaration of Helsinki. DNA extracted from micro-dissected tumour cells of formalin-fixed paraffin-embedded BI-ALCL samples (QIAamp DNA Mini kit; Qiagen, Germantown, MD, USA) was used to prepare DNA libraries (Sureselect kit; Agilent Technologies, Santa Clara, CA, USA). Sequencing was performed on a HiSeq2500 (Illumina, San Diego, CA, USA) using a panel of 465 cancer-associated genes (Table SI). The sequence data were aligned to the human reference genome (hg19) and variants were identified using NextGENe (SoftGenetics, State College, PA, USA). The average read depth of the samples was $400 \times$ (Table SII). Somatic mutations were identified by comparison of variants detected in lymphoma with those from matched constitutional DNA. Common variants ( $>1 \%$ frequency) present in the 1000 genomes database, and the database of Columbia University were removed. Somatic mutations were classified using the prior literature, and two different prediction algorithms (SIFT http://sift.bii.a-star.edu.sg and Polyphen-2 [PP2] http://genetics.bwh.harvard.edu/pph2/). The exonic somatic variants were confirmed by bidirectional Sanger sequencing using Big-Dye terminators v3.1 (Applied Biosystems, Carlsbad, CA, USA).

The clinical and pathological features of the patients are summarized in Table I. Informative results were obtained in five of seven cases (Table SII); analysis failed in two cases due to the poor quality of DNA. Five somatic variants affecting four genes were identified in two cases: one intronic and four within coding regions (Fig 1 and Table SIII).

A STAT3 missense variant (p.S614R) affecting the SH2 domain, which mediates STAT3 dimerization, was detected in one of these two BI-ALCLs. JAK/STAT signalling is implicated in cell proliferation, differentiation and apoptosis, and aberrant activation of STAT3 has been reported in several human cancers associated with persistent immune stimulation and/or inflammation. Notably, the gain-of-function mutation (S614R) was recently described in one BI-ALCL (Blombery et al, 2016), and has been reported in angioimmunoblastic $\mathrm{T}$ cell lymphomas, chronic lymphoproliferative disorders of natural killer cells, and T-cell large granular lymphocyte leukaemias (Odejide et al, 2014). Moreover, gain-of-function mutations in STAT3 have been reported in $18 \%$ of systemic ALK-negative ALCLs and 5\% of cutaneous ALCLs (Crescenzo et al, 2015). An in vitro study using BI-ALCL-derived cell lines also showed activation of the JAK/STAT pathway through autocrine production of interleukin 6, suggesting a possible pathogenic mechanism (Lechner et al, 2012).

A frameshift deletion causing a premature stop codon in SOCS1 (p.P83Rfs*20) was detected in the BI-ALCL harbouring the STAT3 mutation. SOCS1 is a negative feedback regulator of the JAK/STAT pathway. The p.P83Rfs*20 mutation deletes the C-terminal SOCS box domain and partially deletes the $\mathrm{SH} 2$ domain, which downregulates the kinase activity of JAK. Loss-of-function mutations of SOCS1, leading to constitutive activation of JAK/STAT signalling, have been described in B-cell lymphomas and in classical Hodgkin lymphomas (Mottok et al, 2009). Moreover, SOCS1 was found to be silenced by miR-155 in ALK-negative ALCL (Merkel et al, 2015). Mutations in STAT3 and SOCS1 suggest that deregulated activation of the JAK/STAT pathway may contribute to the development of BI-ALCL. 


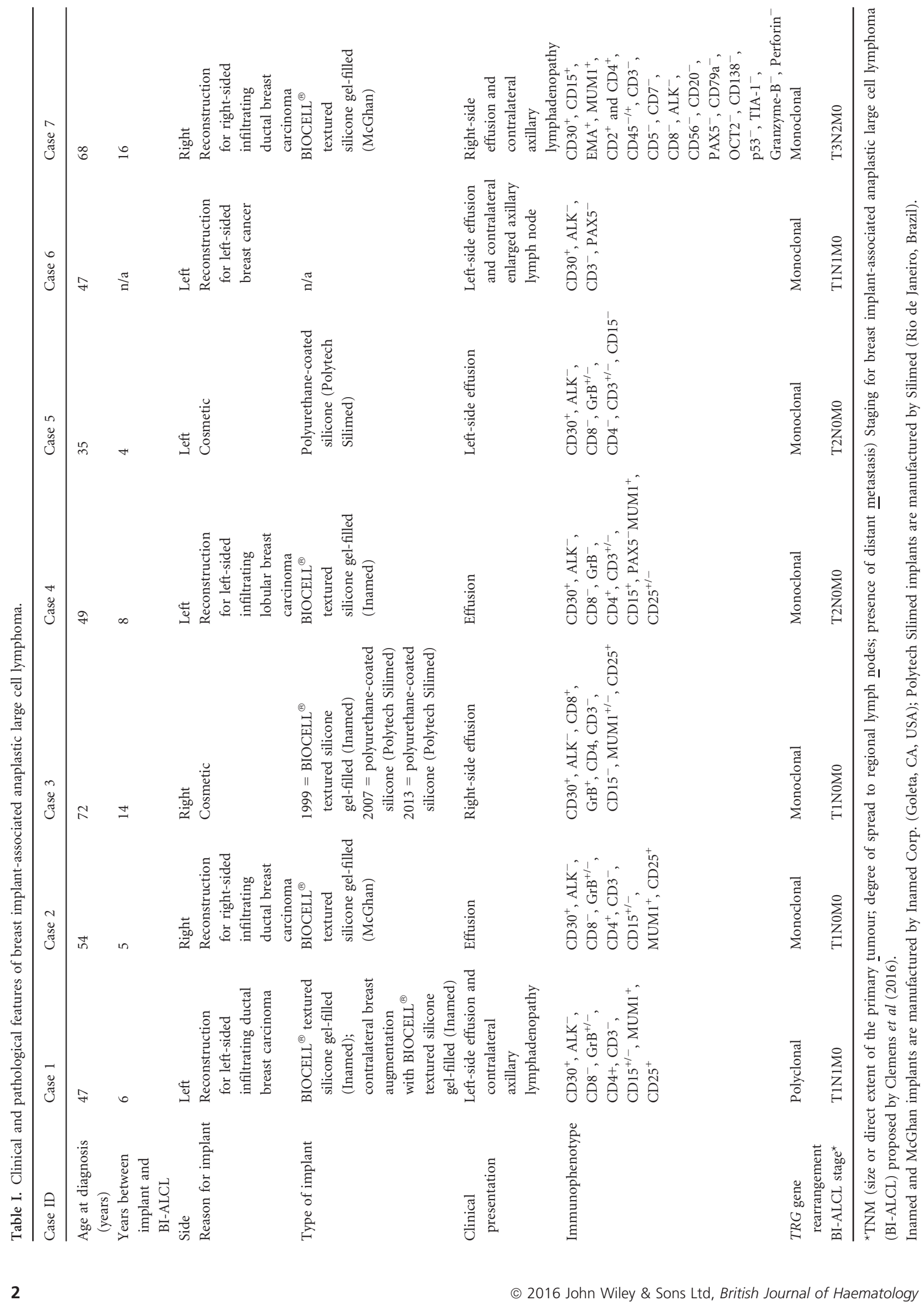




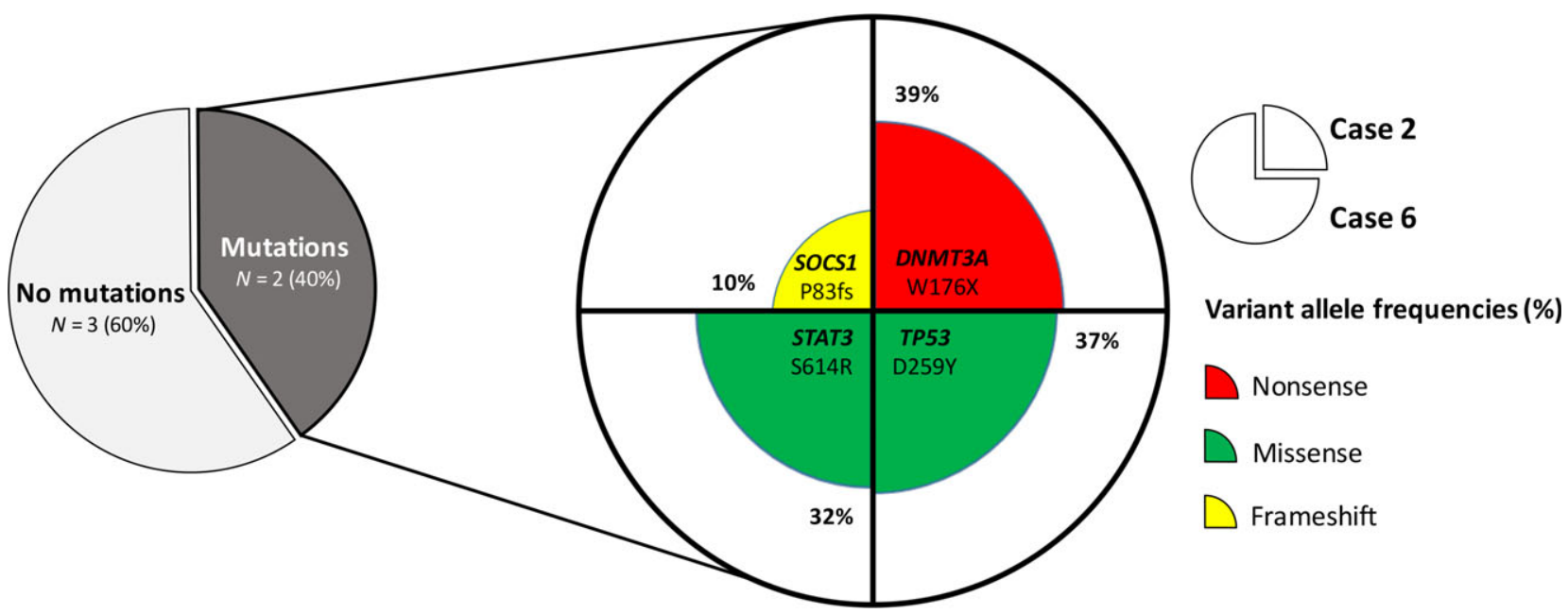

Fig 1. Somatic mutation frequency and spectrum in five breast implant-associated ALK-anaplastic large cell lymphomas evaluated by targeted next-generation sequencing. Exonic variants affecting four genes were identified in two out of five cases (Cases 2 and 6, Table I). In Case 2 a nonsense mutation of DNMT3A was detected, while Case 6 harboured missense mutations in STAT3 and TP53 as well as a frameshift mutation in SOCS1 (variant allele frequencies are shown in each quadrant).

A missense mutation of TP53 (p.D259Y) affecting the DNA binding domain was also observed in the aforementioned case. Although TP53 mutations are uncommon in peripheral T-cell lymphomas (PTCLs) (Odejide et al, 2014), p.D259Y has been documented in several solid tumours (Petitjean et al, 2007).

The second BI-ALCL case with a somatic variant harboured a nonsense mutation in DNMT3A (p.W176X). DNMT3A is a DNA methyltransferase required for genome-wide de novo methylation. Mutations in DNMT3A, mostly impairing DNMT3A catalytic activity, have been reported in $8-22 \%$ of myeloid neoplasms and 33\% of PTCLs (Odejide et al, 2014; Yang et al, 2015). Recent studies have identified DNMT3A mutations in pre-leukaemic haematopoietic stem cells (HSCs) within the founding clones of acute myeloid leukaemias (Yang et al, 2015), in CD $34^{+}$progenitors of T-cell lymphomas (Odejide et al, 2014) and in $16 \%$ of early T-cell precursor acute lymphoblastic leukaemias (ALLs), a subgroup of T-lineage ALLs characterized by specific stem cell and myeloid features (Yang et al, 2015). It has been speculated that DNMT3Amutant HSCs are predisposed to undergo neoplastic transformation upon acquiring mutations in other genes, which cooperate with DNMT3A mutations, fostering the development of distinct haematological malignancies. Our finding suggests that DNMT3A mutations in BI-ALCL might contribute to malignancy by deregulating the DNA methylation landscape of neoplastic T-cells or their precursors.

No somatic mutations were identified in three out of five evaluable cases. Nevertheless, it remains plausible that, similar to observations in systemic and cutaneous ALCL (Crescenzo et al, 2015), deregulation of the JAK/STAT pathway via the generation of fusion transcripts might occur in subsets of non-mutated BI-ALCLs.
Altogether our results confirm that mutations in JAKSTAT pathway genes occur in BI-ALCL, with the identification of SOCS1 mutation for the first time in this disease and TP53 and DNMT3A mutations as additional somatic events. Our findings add to the nascent literature regarding the genetic alterations underlying BI-ALCL and suggest further exploration of the involved molecular pathways in this malignancy.

\section{Authorship contributions}

A.D.N. and G.B. designed experiments, analysed the data and wrote the paper; M.M., P.J. and E.M. analysed the data and wrote the paper; E.D. performed experiments; W.A. performed bioinformatic analysis, A.D.N., G.B., B.A., F.F., E.M. and F.S.D.P. collected the samples and reviewed histology.

\section{Conflict-of-interest disclosure}

All the authors declare that they have no conflict of interest.

\author{
Arianna Di Napoli ${ }^{1}$ \\ Preti Jain ${ }^{2}$ \\ Enrico Duranti ${ }^{1}$ \\ Elizabeth Margolskee ${ }^{2}$ \\ Walter Arancio ${ }^{3}$ \\ Fabio Facchetti ${ }^{4}$ \\ Bachir Alobeid ${ }^{2}$ \\ Fabio Santanelli di Pompeo 5 \\ Mahesh Mansukhani ${ }^{2}$ \\ Govind Bhagat ${ }^{2}$ \\ ${ }^{1}$ Department of Clinical and Molecular Medicine, Sant'Andrea Hospital, \\ Sapienza University, Rome, Italy, ${ }^{2}$ Department of Pathology and Cell
}




\section{Correspondence}

Biology, Columbia University Medical Center, New York Presbyterian Hospital, New York, NY, USA, ${ }^{3}$ Tumour Immunology Unit, Human Pathology Section, Department of Health Science, Palermo University, Palermo, ${ }^{4}$ Pathology Section, Department of Molecular and Translational Medicine, University-Spedali Civili of Brescia, Spedali Civili di Brescia, Brescia, and ${ }^{5}$ Plastic Surgery Unit, Sant'Andrea Hospital, Sapienza University, Rome, Italy.

E-mails:arianna.dinapoli@uniroma1.it; gb96@cumc.columbia.edu

Keywords: breast implant-associated anaplastic large-cell lymphoma, STAT3, DNMT3A, TP53, SOCS1, somatic mutations

\section{Supporting Information}

Additional Supporting Information may be found in the online version of this article:

Table SI. Genes included in targeted next-generation sequencing panel.

Table SII. Coverage statistics for targeted next-generation sequencing assay.

Table SIII. Somatic variants in BI-ALCL.

\section{References}

Blombery, P., Thompson, E., Jones, K., Mir Arnau, G., Lade, S., Markham, J.F., Li, J., Deva, A., Johnstone, R.W., Khot, A., Prince, H.M. \& Westerman, D. (2016) Whole exome sequencing reveals activating JAK1 and STAT3 mutations in breast implant-associated anaplastic large cell lymphoma anaplastic large cell. Haematologica, 101, e387-e390.

Clemens, M.W., Medeiros, L.J., Butler, C.E., Hunt, K.K., Fanale, M.A., Horwitz, S., Weisenburger, D.D., Liu, J., Morgan, E.A., Kanagal-Shamanna, R., Parkash, V., Ning, J., Sohani, A.R., Ferry, J.A., Mehta-Shah, N., Dogan, A., Liu, H., Thormann, N., Di Napoli, A., Lade, S., Piccolini, J., Reyes, R., Williams, T., McCarthy, C.M., Hanson, S.E., Nastoupil, L.J., Gaur, R., Oki, Y., Young, K.H. \& Miranda, R.N. (2016) Complete surgical excision is essential for the management of patients with breast implant-associated anaplastic large-cell lymphoma. Journal of Clinical Oncology, 34, 160-168.

Crescenzo, R., Abate, F., Lasorsa, E., Tabbo', F., Gaudiano, M., Chiesa, N., Di Giacomo, F., Spaccarotella, E., Barbarossa, L., Ercole, E., Todaro, M., Boi, M., Acquaviva, A., Ficarra, E., Novero, D., Rinaldi, A., Tousseyn, T., Rosenwald, A., Kenner, L., Cerroni, L., Tzankov, A.,
Ponzoni, M., Paulli, M., Weisenburger, D., Chan, W.C., Iqbal, J., Piris, M.A., Zamo', A., Ciardullo, C., Rossi, D., Gaidano, G., Pileri, S., Tiacci, E., Falini, B., Shultz, L.D., Mevellec, L., Vialard, J.E., Piva, R., Bertoni, F., Rabadan, R. \& Inghirami, G. (2015) Convergent mutations and kinase fusions lead to oncogenic STAT3 activation in anaplastic large cell lymphoma. Cancer Cell, 27, 516-532.

Lechner, M.G., Megiel, C., Church, C.H., Angell, T.E., Russell, S.M., Sevell, R.B., Jang, J.K., Brody, G.S. \& Epstein, A.L. (2012) Survival signals and targets for therapy in breast implant-associated ALK-anaplastic large cell lymphoma. Clinical Cancer Research, 18, 4549-4559.

Merkel, O., Hamacher, F., Griessl, R., Grabner, L., Schiefer, A.I., Prutsch, N., Baer, C., Egger, G., Schlederer, M., Krenn, P.W., Hartmann, T.N., Simonitsch-Klupp, I., Plass, C., Staber, P.B., Moriggl, R., Turner, S.D., Greil, R. \& Kenner, L. (2015) Oncogenic role of miR-155 in anaplastic large cell lymphoma lacking the $t$ (2;5) translocation. Journal of Pathology, 236, 445-456.

Mottok, A., Renné, C., Seifert, M., Oppermann, E., Bechstein, W., Hansmann, M.L., Küppers, R. \& Bräuninger, A. (2009) Inactivating SOCS1 mutations are caused by aberrant somatic hypermutation and restricted to a subset of Bcell lymphoma entities. Blood, 114, 4503-4506.

Odejide, O., Weigert, O., Lane, A.A., Toscano, D., Lunning, M.A., Kopp, N., Kim, S., van Bodegom, D., Bolla, S., Schatz, J.H., Teruya-Feldstein, J., Hochberg, E., Louissaint, A., Dorfman, D., Stevenson, K., Rodig, S.J., Piccaluga, P.P., Jacobsen, E., Pileri, S.A., Harris, N.L., Ferrero, S., Inghirami, G., Horwitz, S.M. \& Weinstock, D.M. (2014) A targeted mutational landscape of angioimmunoblastic T-cell lymphoma. Blood, 123, 1293-1296.

Petitjean, A., Mathe, E., Kato, S., Ishioka, C., Tavtigian, S.V., Hainaut, P. \& Olivier, M. (2007) Impact of mutant p53 functional properties on TP53 mutation patterns and tumor phenotype: lessons from recent developments in the IARC TP53 database (database version R18, April 2016). Human Mutation, 28, 622-629.

Swerdlow, S.H., Campo, E., Pileri, S.A., Harris, N.L., Stein, H., Siebert, R., Advani, R., Ghielmini, M., Salles, G.A., Zelenetz, A.D. \& Jaffe, E.S. (2016) The 2016 revision to the World Health Organization classification of lymphoid neoplasm. Blood, 127, 2375-2390.

Yang, L., Rau, R. \& Goodell, M.A. (2015) DNMT3A in haematological malignancies. Nature Reviews Cancer, 15, 152-165. 